\title{
Viscous flow around spherical particles in different arrangements
}

\author{
Tsvetan Kotsev* \\ Sofia University, Department of Mathematics and Informatics, James Boucher blvd. No. 5, \\ 1014 Sofia, Bulgaria
}

\begin{abstract}
Viscous flow past two or more spherical particles in direction parallel or perpendicular to the line connecting the body centers is studied numerically at Reynolds number up to 200 . The FEM is used to simulate the flow structure for different arrangements and diameters of the spheres. The drag and lift coefficients are calculated as functions of the flow velocity and the distance between the spheres. The results show the downstream spheres face a reduced drag compared to a single sphere and that the upstream one changes its drag coefficient according to the distance between the bodies. In the case of side by side arranged spheres, an effect of repulsion exists up to a certain separation distance and after that a weak pulling effect appears. A configuration of three (or more) spherical particles arranged in " $\mathrm{V}^{\text {“ }}$ formation was examined where one body is set to be a leading and the others are located symmetrically up and down behind it. The flow characteristics are studied when the angle behind the leading sphere vary from $40^{\circ}$ to $180^{\circ}$. This configuration supposes stronger interaction between the bodies due to the influence of the wakes behind the first sphere to the downstream ones and to the lift forces appeared.
\end{abstract}

\section{Introduction}

Investigation of the flow and hydrodynamic interaction between droplets, solid spherical particles is important in various engineering applications and chemical processes, pollution diffusion, energy systems, ship hydrodynamics, etc. Classical problem of Stokes [1] for a single sphere in uniform incompressible flow is the first one among many others that was solved in analytical way when the inertial terms are neglected but relatively less are the studies investigating the flow past two or more particles. Stimson and Jeffrey [2] have studied the flow past two spheres that are close together. Later Kim [3] has suggested a technique for multi-sphere Stokes flow problems that involves the analytical transformation of basic functions between coordinates based on different spheres. An analytical study of hydrodynamic interaction of three spheres in a quiescent viscous fluid was considered by Wilson [4] for spheres with equal and different diameters using a method based on Lamb's solution to Stokes flow and the method of reflections. Fluid dynamic interaction between two spheres in tandem configuration was experimentally studied by Tsuji, Morikawa \&Terashima [5] for Reynolds numbers between 100 and 1000 and Zhu, Liang \& Fan [6] for Re varying from 20 to 130. They have investigated the evolution of the flow field and drag experienced by the bodies when the distance between them vary. Chen and $\mathrm{Wu}[7]$ studied the flow characteristics between the

\footnotetext{
* Corresponding author: tkotsev@fmi.uni-sofia.bg
} 
two spheres experimentally at Reynolds numbers in the range of $54 \leq \mathrm{Re} \leq 118$. Applications of computational fluid dynamics (CFD) have widely increased in the field of heat and fluid flow. The benefits of CFD compared to the experimental methods are that simulation is much cheaper and not time consuming. For moderate Reynolds numbers where both the viscous and convective terms must be taken into consideration the only way to receive an approximately good solution is the numerical calculations and in the literature exist such investigations but as a whole they are not so many especially for complex body configurations. Three-dimensional flow over two spheres placed side-by-side was investigated by Kim et al. [8] at $\mathrm{Re}=50,100$, and 150 for various separation distances. They considered the lift, moment and drag coefficients on the spheres and investigated the dependence on the distance between the spheres. Schouveiler et al. [9] studied the interactions of the wakes of two spheres placed side-by-side both experimentally and numerically. They applied various separation distances, for example, in the range of $1.0 \leq \mathrm{d} \leq 4,6$ at $\mathrm{Re}=300$ and revealed that for a given Reynolds number, the wake frequency varies smoothly with separation distance.

In the present study the steady flow past two, three and more spherical particles arranged in line or side by side is simulated at Reynolds numbers up to 200. Comparison both with experimental and numerical results was done to test the model. A complex configuration of three and seven spherical particles arranged in , $\mathrm{V}^{\prime}$ formation was examined. The flow characteristics are studied when the angle behind the leading sphere varies from $40^{\circ}$ to $180^{\circ}$. This configuration supposes stronger interaction between the bodies due to influence of the wakes behind first sphere to the downstream ones and to the lift forces appeared.

\section{Statement of the problem and method of solution}

Two or more rigid spherical particles are immersed in a uniform stream of viscous fluid. The velocity of the ongoing fluid is $\mathrm{V}_{0}$ and is parallel or perpendicular to the line connecting the body centers. The fluid is accepted to be water with density of $998\left[\mathrm{~kg} / \mathrm{m}^{3}\right]$ and dynamic viscosity $0,00101[\mathrm{~Pa} . \mathrm{s}]$. The following arrangements of the spheres according to the flow direction are considered:

a) The flow direction is parallel to the line connecting the body centers. Their diameters may be equal or different. In this case the flow is axisymmetric in respect to the central line that allows the three-dimensional problem to be reduced to a two-dimensional one.

b) The flow direction is perpendicular to the line connecting the centers of the spheres (side by side arrangement) that makes the problem three-dimensional.

c) The flow around three or more spheres arranged in , $\mathrm{V}^{\prime}$ formation where one sphere is set to be a leading one and the others are located symmetrically up and down behind it.

It is obvious both from the experiments and numerical simulations that the flow past a single sphere becomes unstable for Re about 200 when the wake starts to oscillate. Here Re is based on the diameter of the upstream sphere $-R e=2 R_{1} \cdot V_{0} / v$, where $v$ is the kinematic viscosity and takes values from 0,01 to 200 . Another parameter having strong influence on the flow picture is the distance between the centers varying here from 0,25 up to 30 times the radius of the upstream sphere. For the purpose of the present calculations a cartesian coordinate system is used with $x$ axis parallel to the line connecting the sphere centers in the axisymmetric case and perpendicular to this line in the case for side by side arranged bodies. Equations of the flow motion are the continuity equation (1) and the momentum equation for steady Newtonian flows (2).

$$
\begin{gathered}
\nabla \cdot \mathbf{v}=0 \\
\rho(\mathbf{v} . \nabla) \mathbf{v}=-\nabla p+\mu \nabla^{2} \mathbf{v},
\end{gathered}
$$

where $\mathbf{v}$ is velocity vector, $\mu-$ dynamic viscosity, $\mathrm{p}$ - pressure, $\rho$ - fluid density. 
Boundary conditions are:

- no slip on the sphere surfaces -

$$
\begin{aligned}
& \mathbf{v}=0 \\
& v=V_{0} \\
& p=0 \\
& \text { v.n }=0 .
\end{aligned}
$$

- zero pressure at the outlet -

The drag and lift forces are calculated integrating the stress tensor on the body surfaces:

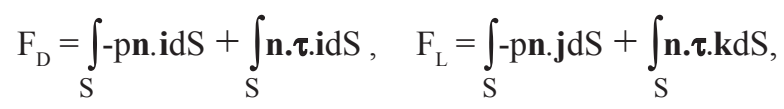

where $\mathrm{S}$ denotes the surface of the spheres, $\mathbf{i}$ and $\mathbf{j}$ are unit vectors in $\mathrm{x}$ and $\mathrm{y}$ direction respectively, $\mathbf{n}$ is the outward normal vector at the surface and $\boldsymbol{\tau}$ is the viscous stress tensor.

Non-dimensional drag and lift coefficients $C_{D}$ and $C_{L}$ are defined as:

$$
\mathrm{C}_{\mathrm{D}}=\frac{F_{D}}{\rho \mathrm{V}_{0}^{2} \mathrm{~A}_{\mathrm{pr}} / 2} \quad \mathrm{C}_{\mathrm{L}}=\frac{F_{L}}{\rho \mathrm{V}_{0}^{2} \mathrm{~A}_{\mathrm{pr}} / 2}
$$

where $A_{p r}$ is the area of the sphere projected on a plane perpendicular to the flow direction. The equations (1) and (2) were solved numerically using COMSOL Multiphysics package based on the finite element method. It supposes covering the computational domain with a mesh of different type of elements. Most used ones are tetrahedral in 3D and triangle in $2 \mathrm{D}$ case because these elements approximate in the best way any complicated geometrical domain. The software gives a possibility to cover with a fine mesh the regions near to the bodies where the gradients of the velocity are significant and with a coarse one the area far from them (Fig.1).

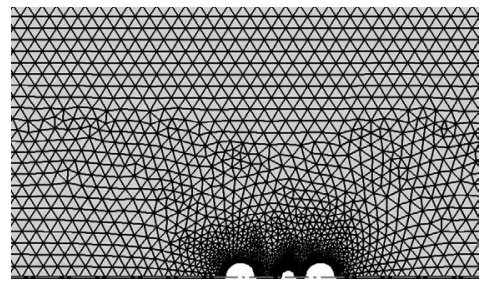

a)

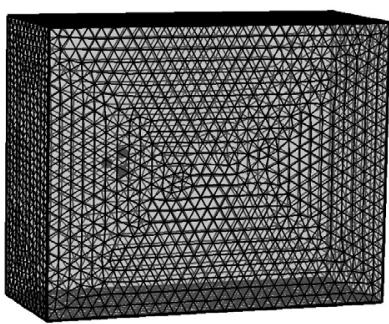

b)

Fig. 1 a) Mesh with triangle elements for 2D. b) Mesh with tetrahedral elements for 3D case.

Taking into account the information about the physics of the process, material of the bodies, properties, boundary conditions, elements type and shape, the software assembles a system of equations which need to be solved to get an answer of the finite element problem. The size of the matrix of this system is the number of degrees of freedom (DOF) of the model, where the number of DOF is a function of the number of elements, the discretization order used in each physics, and the number of variables solved for. For example, in the axisymmetric case of flow past two spheres in line the complete mesh consists of 23352 domain elements and 626 boundary elements and total number of DOF 161370. To solve the system of equations both direct and iterative solvers could be used, this one used here is GMRES - an iterative solver that supposes good convergence, solution time and use of memory. In the case of side by side located spheres an extra fine mesh of elements was used that results in 795537 domain elements, 18346 boundary elements, and 616 edge elements and 551076 DOF. 


\section{Results}

In order to test the model a comparison with the flow around a single sphere was done. For that purpose the distance between two equal spheres in tandem arrangement was set to be 30 times the radius of the upstream sphere, so the interaction between the bodies to be negligible small. At Fig. 2 a) is shown the experimental result [10] for the laminar flow around a single sphere for $\mathrm{Re}=118$. Numerical results for the same Re and spheres far enough one form another show good accordance with the experiment carried out using water and aluminum dust to visualize the flow.

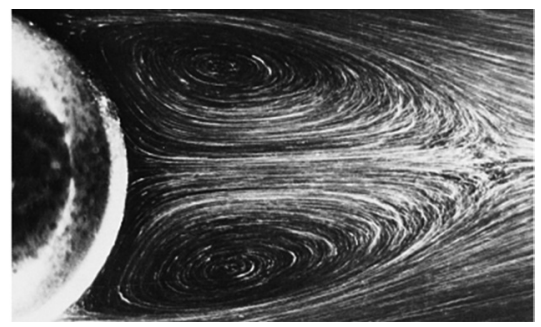

a)

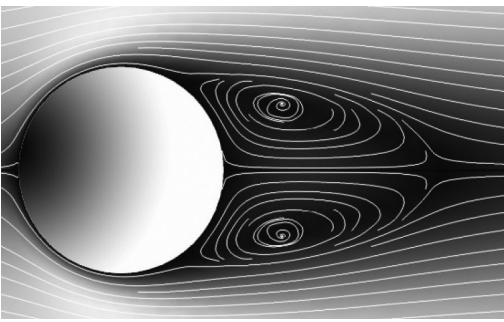

b)

Fig. 2 a) Experimental study of the flow around a single sphere, $R e=118$ b) present calculations

It could be seen that the structure of the recirculating zones behind the spheres is identical and the length of the eddies in both cases is about one sphere diameter. The length of the vorticity zone behind the sphere increases as a logarithm of Reynolds number unlike of the case for a cylinder where the wake grows almost linear with Re. Line representing the drag coefficient of a single sphere measured experimentally for wide range of Re together with Stokes and Oseen's approximations for small Re are shown at Fig.3. The values from the numerical calculations are also noted with black dots. It is seen that our data are in a good accordance with Stokes and Oseen's solutions for small Reynolds numbers as well as with the experimental data for moderate Re.

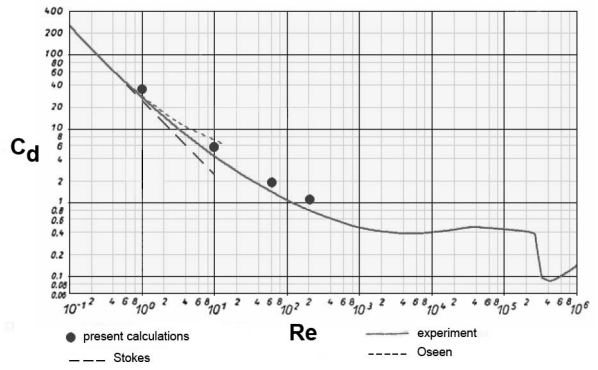

Fig. 3. Comparison with the drag coefficient for a single sphere.

\subsection{Flow past spheres in line arrangement}

\subsubsection{Two equal spheres in tandem}

The flow structure past two spheres in tandem for small and moderate Reynolds numbers was a subject of study both analytically and numerically respectively. Existence of a suitable coordinate system allowing to describe the body surfaces as coordinate lines and developing of the numerical techniques has resulted in many studies - Zapryanov et al. [11], Tal et.al. [12], etc. Here an attempt was done to confirm and expand these results and to demonstrate the efficiency of the FEM integrated in COMSOL package to solve complicated flow problems. 
Numerical simulations were done for two spheres with radius 0,02 [m] and Reynolds numbers from 0,01 to 200 for different distances between the spheres. In all cases considered, the distance between bodies is denoted with $d$ and is normalized by the diameter of the upstream sphere. The flow picture past two equal spheres for distance between them equal to 1 diameter is shown at Fig. 4. Up to $\mathrm{Re}=10$ the flow is similar to Stokes one - there is no separation of the fluid from the surfaces and no forming of vortices behind the bodies. Experiments show that for a single sphere separation of the flow from the surface begins at Re about 25 . Here due to a presence of a second body in the wake behind the upstream one the separation from the first sphere appears at lower values of $\mathrm{Re}-$ about 20 .

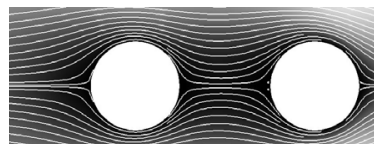

$\mathrm{Re}=10$

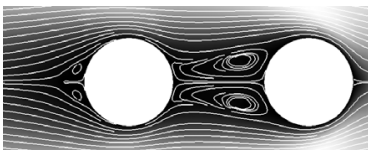

$\mathrm{Re}=47$

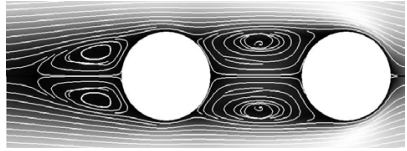

$\mathrm{Re}=200$

Fig. 4. Flow around two equal spheres at $\mathrm{d}=1$.

The point of separation is at first close to the rear of the sphere and separation results in the formation of a ring eddy attached to the rear surface of the sphere. As Re increases the point of separation moves up to the side of the sphere and the wake in the gap grow and enlarges toward the second body. At $\mathrm{Re}=30$ a small vortex is seen in front of the downstream sphere that merge with this one behind the upstream body when the velocity of the fluid grows. There is no vortex zone behind the downstream sphere, it appears at Re about 40. If the distance between spheres decreases to 0,5 the mention above events begin earlier - separation of the fluid from upstream sphere is visible at $\mathrm{Re}=10$ and the vortex zone in the gap is well formed for lower Reynolds number $-\mathrm{Re}=25$. When the two spheres touch each other, the total drag of this assemblage is less than the drag of a single sphere but this depends on Re and is connected with the flow structure. For example, at $\mathrm{Re}=10$ there is no eddy behind the downstream body and the total drag of two touching spheres is bigger than the drag of a single sphere. At $\mathrm{Re}=62$ when vorticity behind the second body is well formed, then the total drag becomes less than the drag of a single body. This effect disappears for distance more than 0,21 and the sum of the drag of two bodies becomes higher than this one of a single one. The distance between the bodies at which the total drag is less that this one of a single body grows with $\mathrm{Re}$ and at $\mathrm{Re}=200$ it is appr. 0,5 but the most important factor that influences the hydrodynamic interaction of the particles is the distance between them.

The drag coefficient of the spheres with respect to Reynolds number is shown at Fig.5.

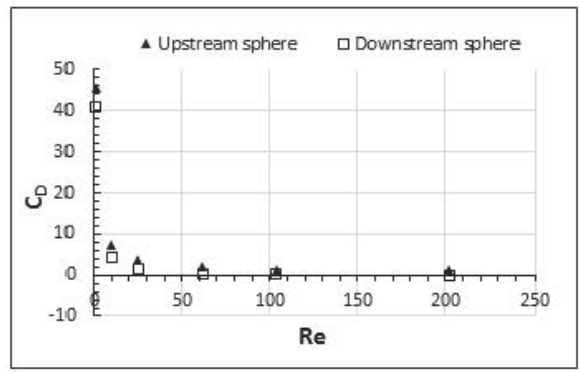

Fig. 5 Drag coefficient of two equal spheres at $\mathrm{d}=1$ vs Reynolds number.

The downstream sphere faces a reduced drag compared to an upstream one. It decreases rapidly with $\mathrm{Re}$, becomes close to zero and for $\mathrm{Re}=200$ is negative even with a small value that means there is a weak attractive effect between bodies. The upstream sphere also changes its 
drag coefficient - it decreases or increases by approximately $12 \%$ according to the drag of a single sphere and depends strongly on the distance between the spheres.

\subsubsection{Two different spheres in tandem}

A case when the upstream sphere is bigger than the downstream one is considered. The radius of the first sphere is $0,02[\mathrm{~m}]$, this one of the second is twice less $-0,01[\mathrm{~m}]$. A flow picture for $\mathrm{Re}=62$ and $\mathrm{d}=0$ and $\mathrm{d}=0,5$ is shown at Fig. 6 . Like in the case of equal spheres at distance $d=0,5$ the vortex zone behind the bigger upstream sphere appears for Re about 10, but separation from the downstream one could be seen for quite higher values of Reynolds number $-\operatorname{Re} \approx 90$.

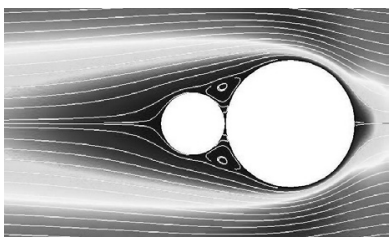

a)

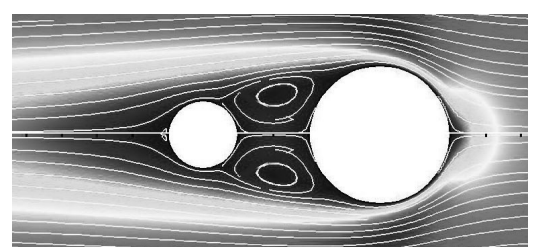

b)

Fig. 6. Flow past two different spheres a) $d=0$, b) $d=0,5$

To follow the dependency of the drag coefficient with Reynolds number calculations were done for $\mathrm{Re}=10,25,62,100$ and 200 for distances between the spheres equal to 0,5 and 1 (Fig. 7a). The drag of the upstream body falls down with Re but stays almost the one and the same both for $\mathrm{d}=0,5$ and 1 at all values of Re considered. The drag coefficient of the downstream sphere also decreases with $\mathrm{Re}$ and for values of $\mathrm{Re}>50$ and $\mathrm{d}=0,5$ becomes negative that indicates attractive force between bodies. The interaction between different spheres with distance $\mathrm{d}$ for fixed Reynolds number $(\mathrm{Re}=62)$ is shown at Fig. $7 \mathrm{~b}$ ) where $\mathrm{d}$ takes values 0 , $0,5,1,1,5$ and 2,5 . For comparison is drawn also the curve representing the drag coefficients of two equal spheres for the same parameters. When the two spheres touch each other the total drag analogous to the case of equal spheres is also less than the drag of a single sphere.

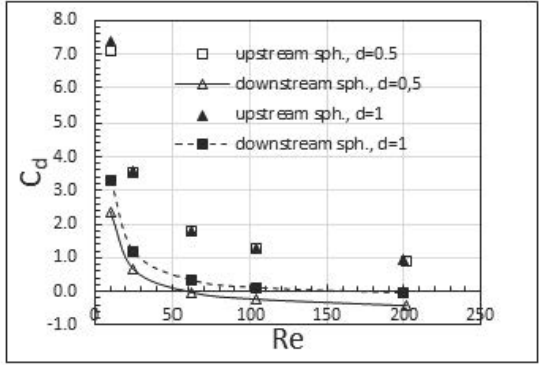

a)

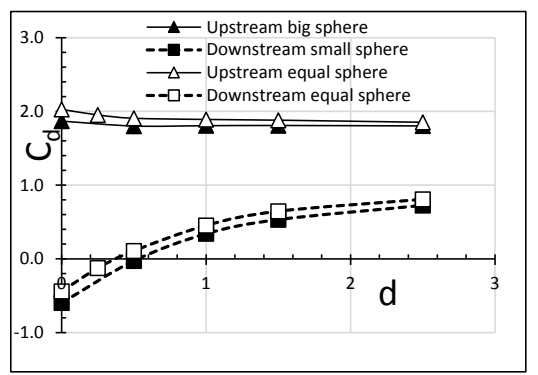

b)

Fig. 7 Drag coefficient vs Re and d of two different spheres: a) $d=0,5 ; 1$ b) $\operatorname{Re}=62, d=0 ; 0,5 ; 1 ; 1,5 ; 2,5$

The distance between spheres up to which this trend is valid for $\mathrm{Re}=62$ is appr. $\mathrm{d}=0,5$ while for the case of equal spheres this distance is appr. twice less. The drag coefficient of the first sphere is positive and decreases slightly with $d$ while this one for the second sphere stays negative up to $d=0,5$. With further taking away the second body, $C_{D 1}$ is almost constant while $\mathrm{C}_{\mathrm{D} 2}$ increases and takes positive values. 


\subsubsection{Three equal spheres in line}

If we denote with $d_{12}$ the distance between upstream and middle sphere and with $d_{23}$ - the distance between the middle and downstream sphere, the following cases are investigated:

\begin{tabular}{|c|c|c|c|c|c|c|}
\hline $\mathrm{d}_{12}$ & 0 & 0 & 0 & 1 & 0.5 & 1 \\
\hline $\mathrm{d}_{23}$ & 0 & 0.5 & 1 & 1 & 0 & 0 \\
\hline
\end{tabular}

Fig. 8 shows the flow at $R e=62$ and two distances between bodies $-\mathrm{d}_{12}=\mathrm{d}_{23}=0$ and $\mathrm{d}_{12}=\mathrm{d}_{23}=1$. In the gaps between spheres recirculating zones exists even for small Re, but this one behind the downstream body appears at Re about 42 that is almost twice bigger compared to the flow around a single sphere.

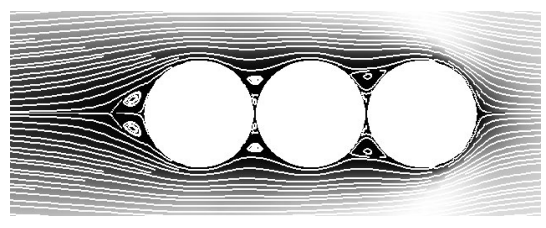

a)

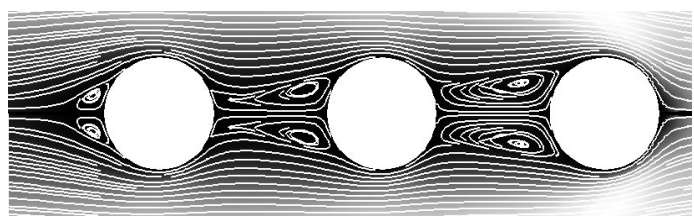

b)

Fig. 8. Flow past 3 equal spheres for $\operatorname{Re}=62$ : a) touching spheres $b) d_{1}=d_{2}=1$ diameter

The variation of the drag coefficients with ratio $\mathrm{k}=\mathrm{d}_{12} / \mathrm{d}_{23}$ and for $\mathrm{Re}=62$ is shown at Fig. 9a). When $\mathrm{k}=1$ all spheres are at one and the same distances each from another. Moving the middle sphere towards the first one its drag coefficient rapidly decrease while the drag coefficient of the upstream sphere increase with appr. $9 \%$. At a distance between upstream and middle sphere about $0,25(\mathrm{k}=0,14)$ the drag coefficient of the middle body takes negative values. If the middle body is moving toward the downstream one $(\mathrm{k}>1) \mathrm{C}_{\mathrm{D} 1}$ keeps almost one and the same value while the drag coefficient of the downstream body quickly fall down but is positive even when second sphere touch the third one.

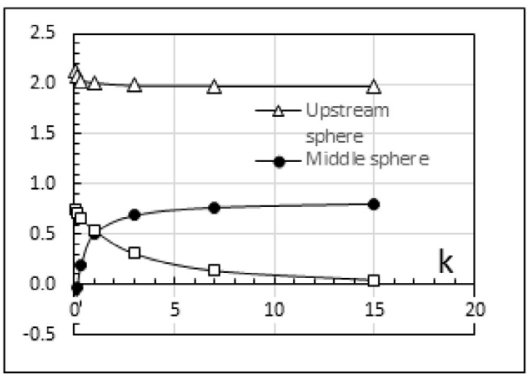

a)

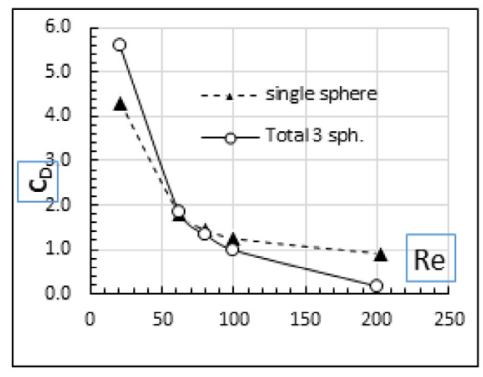

b)

Fig. 9. Drag coefficient of three equal spheres: a) $R e=62$ b) $v s k=d_{12} / d_{23}$.

Three touching spheres feel less drag than a single sphere with the same diameter for Reynolds numbers higher than 60 (Fig.9b).

\subsubsection{Three different spheres in line}

The case when upstream and downstream spheres have equal diameters and are placed at a distance $\mathrm{d}=3$ is considered. The middle body has twice less diameter and changes its position. Initially it touches the upstream sphere, then starts to move towards the downstream one. The flow picture is shown at Fig. 10 for $\mathrm{Re}=62$. 


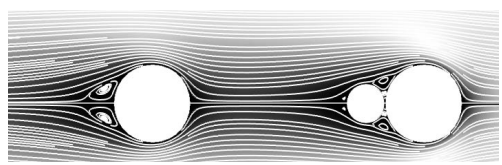

a)

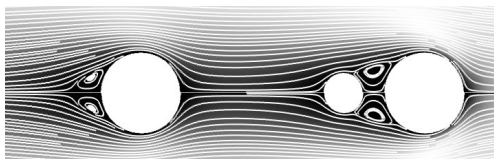

b)

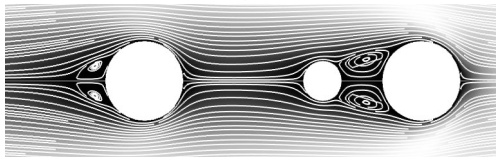

c)

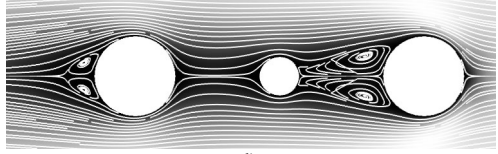

d)

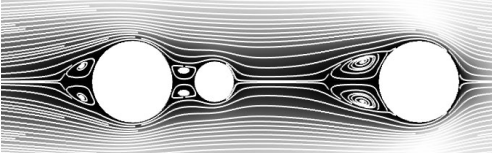

e)

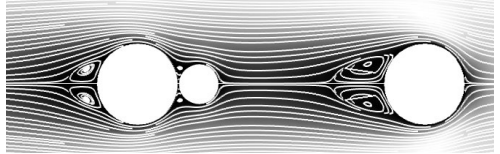

f)

Fig. 10. Flow past three different spheres at $\mathrm{Re}=62$ and various $\mathrm{k}=\mathrm{d}_{12} / \mathrm{d}_{23}$. a) $\mathrm{k}=0, \mathrm{~b}) \mathrm{k}=0,14$, c) $\mathrm{k}=0,33$, d) $\mathrm{k}=1$, e) $\mathrm{k}=7$, f) sphere 2 touch sphere 3

Fig.11 shows the variation of the drag coefficients with Reynolds number and with ratio $\mathrm{k}$ at fixed $\mathrm{Re}=62$. They decrease with increasing of $\mathrm{Re}, \mathrm{C}_{\mathrm{D} 1}$ and $\mathrm{C}_{\mathrm{D} 3}$ stay positive while $\mathrm{C}_{\mathrm{D} 2}$ accept negative values for Reynold numbers higher than 55 .
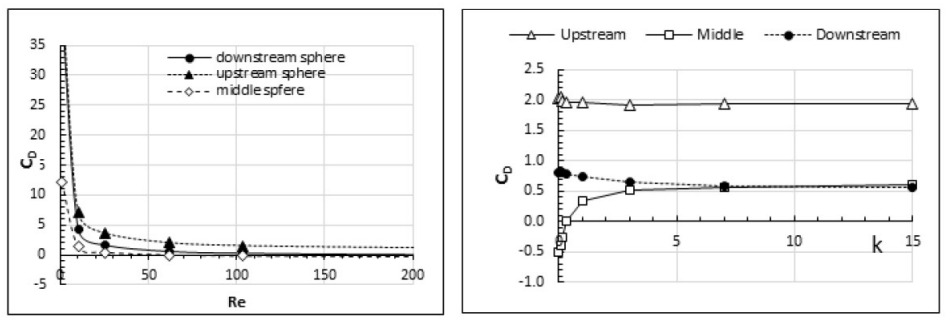

Fig. 11. Drag coefficient of three different spheres vs Re for fixed distance $d_{12}=d_{23}=0,5$.

When $\mathrm{k}=0$ (upstream and middle sphere are touching) the upstream sphere feels almost the same drag as the corresponding one in the case of equal spheres $-\mathrm{C}_{\mathrm{D} 1}$ is 2,038 in the case of different spheres and 2,12 in the case of equal bodies. Increasing $\mathrm{k}$, the drag falls very slow and keeps almost a constant value. The drag coefficient of the downstream sphere $\mathrm{C}_{\mathrm{D} 3}$ decreases with $\mathrm{k}$ and for $\mathrm{k}>7$ takes almost a constant value of 0,5 as opposed when bodies have equal dismeters where $C_{D 3}$ takes positive values close to zero. Quite different is the behavior of the drag of the middle sphere. Being twice smaller than the other two ones it has negative $\mathrm{C}_{\mathrm{D} 2}$ for higher valuse of $\mathrm{k}$ compared with the previous case. $\mathrm{C}_{\mathrm{D} 2}$ is negative up to $\mathrm{k}=0,33$ ( 1 radius between spheres 1 and 2) while for the case of equal spheres the drag coefficient takes negative values up to $\mathrm{k}=0,14$. In other words, the smallest it is the middle sphere, the longer is the distance from the upstream one at which $\mathrm{C}_{\mathrm{D} 2}<0$.

\subsection{Flow past spheres in side by side arrangement}

\subsubsection{Two side by side spheres}

A steady 3D incompressible laminar flow past two identical spheres held fixed with the line connecting the sphere centers normal to a uniform stream was considered. The simulation of the flow was done for $\mathrm{Re}=10,50,62,200$ and separation distances normalized by the 
sphere diameter from 0 (touching spheres) up to 12 . The hydrodynamic interaction in this case strongly depends on the separation distance and different flow regimes could be seen past the bodies. In the case when the spheres touch each other, only one single wake is visible behind them (Fig. 12a). When the separation distance is small the wakes behind each sphere interact strongly and the combined wake is offset with respect to the center plane between the spheres.

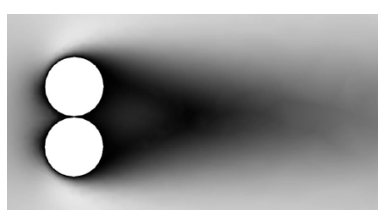

a)

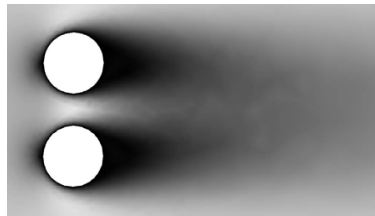

b)

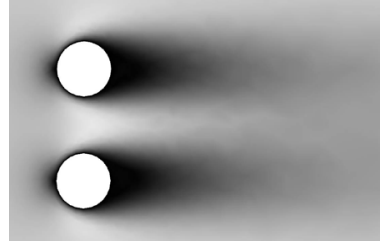

c)

Fig. 12 Flow picture at $R e=50$ : a) $d=0$, b) $d=0,5$, c) $d=1$

This trend continues up to separation distance about 3 when the wakes are almost independent each other. Comparison for the drag coefficients with results of Folkersma et all [12] was done at $\mathrm{Re}=10$ and 50 and for a separation distance $\mathrm{d}=0,5$. Results in [12] are 4,87 and 1,74 respectively while our values are 4,618 and 1,73 . The difference is about $5 \%$ at $\operatorname{Re}=10$ and less than $1 \%$ at $\mathrm{Re}=50$. For separation distances less than $10-10,5$ and $\mathrm{Re}=50$ the lift coefficient is negative that means there is a repulsion of the two spheres. For bigger separation distances the lift coefficient is positive but relatively small that means there is a weak attractive force between the spheres (Fig.13). Further increasing the separation distance leads to a negligible small interaction between spheres.

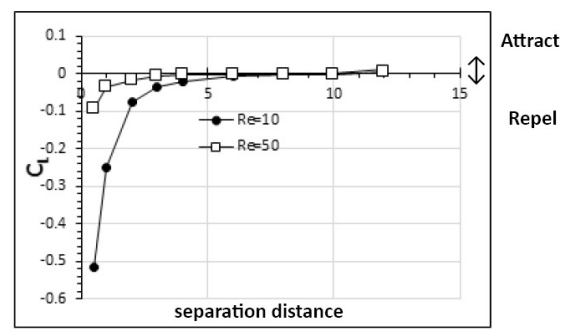

Fig.13. Lift coefficient of two side by side spheres for $\mathrm{Re}=10$ and 50 vs separation distance

\subsubsection{Spheres in „V“ formation}

This configuration of three or more bodies could be seen in different devices in chemical industry, particle suspension flows, fluidized beds, heat exchangers, etc. Of interest in this study is the flow patterns around three and seven equal spheres arranged in a triangular formation. Let us agree the sphere that is in the leading position according to the incoming flow to call leading sphere and to denote it with number 1 . The other spheres let be marked with numbers up to 3 or 7 . We consider the cases when the distance $d$ between the leading sphere and other ones are equal and could vary from 0 to 7 diameters. Reynolds numbers considered are $\mathrm{Re}=10$ and 50. Let the angle between the lines connecting the centers of the upper and lower spheres denote with $\alpha$ and to consider values of $\alpha$ from $30^{\circ}$ to $180^{\circ}$. When all spheres are in contact there is only an one wake behind them (Fig.14). The total drag of this assemblage is 2,562 and is bigger than the drag of a single sphere $-2,116$. For $\alpha=45^{\circ}$ the wake is still one and the interaction of the bodies is significant. Increasing the angle wakes behind each sphere is formed and the mutual influence decrease. 


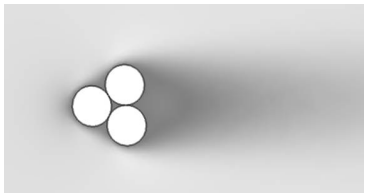

a)

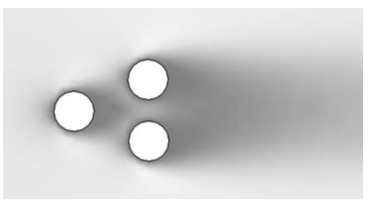

b)

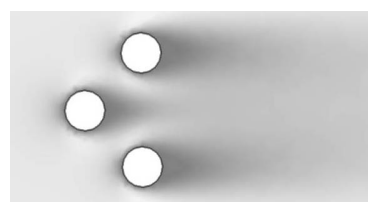

c)

Fig. 14 Flow past three spheres at $\mathrm{Re}=50$ : a) $\mathrm{d}_{12}=\mathrm{d}_{13}=0$; b) $\mathrm{d}_{12}=\mathrm{d}_{13}=1, \alpha=45^{\circ}$; c) $\mathrm{d}_{12}=\mathrm{d}_{13}=1, \alpha=90^{0}$

Lift and total drag coefficients are shown at Fig. 15.
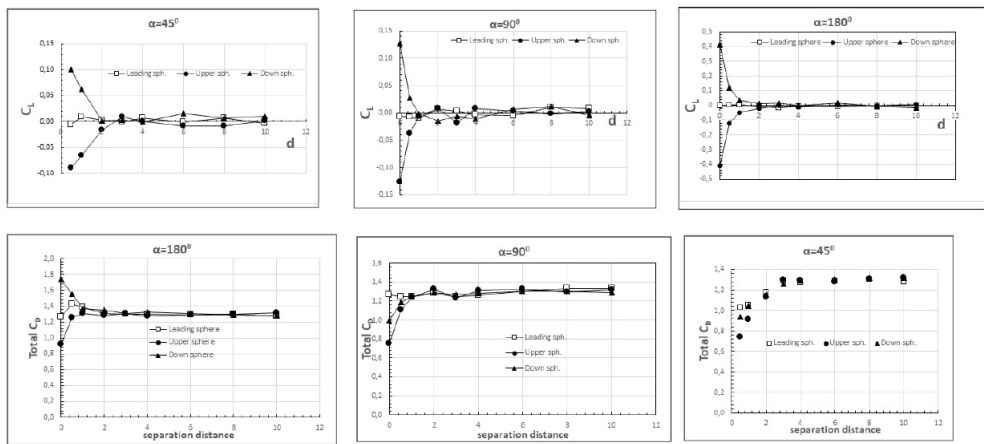

Fig. 15 Lift and total drag coefficient for $\mathrm{Re}=50$ and $\alpha=45^{\circ}, 90^{\circ}$ and $180^{\circ}$

The configuration of seven spheres with equal diameters was chosen to analyze and verify the influence between the wakes formed by the particles. Such a configuration could be related with the well known „V" formation of the birds, which in accordance with the scientists, reduces the drag force that each bird experiences compared to if it were flying alone. The spheres were arranged in different configurations by changing the angle $\alpha$. It takes values $40^{\circ}$, $60^{\circ}, 90^{\circ}, 110^{\circ}, 130^{\circ}, 150^{\circ}$ and $180^{\circ}$. The distances between the spheres are equal to $\mathrm{d}=1$ and $\mathrm{Re}=100$ for all simulations. Due to a flow symmetry the spheres with numbers $1,3,5$ and 7 where chosen to be analyzed. The pictures at Fig. 16 shows the flow in the center plane of the configuration for different angles $\alpha$.

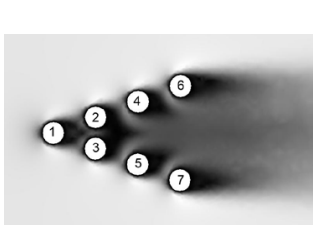

$\alpha=40^{0}$

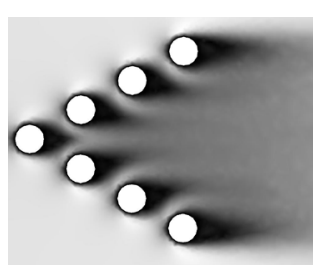

$\alpha=60^{\circ}$

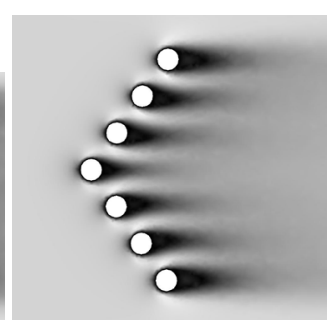

$\alpha=110^{0}$

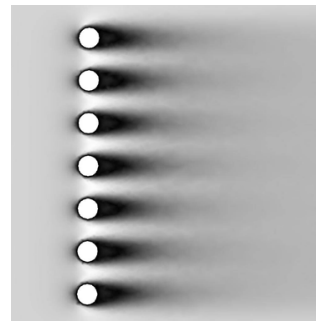

$\alpha=180^{\circ}$

Fig. 16 Flow past 7 spheres at $\mathrm{Re}=100$ and different values of $\alpha$.

When the angle $\alpha$ is small the wakes behind spheres are not formed well except these ones at the end of the formation. As a whole the wake behind this assemblage looks like a wake behind a single delta body immersed in the fluid. With increase of $\alpha$ more and more clearly visible are the individual wakes behind each sphere that interact strongly each other and as a result the drag force coefficients change. The drag coefficients for the spheres 1, 3, 5 and 7 divided by the value of a single sphere are presented in Fig. 17 for $\operatorname{Re}=100$. For $40^{\circ}<\alpha<180^{\circ}$ all spheres have drag coefficients lower than for a single sphere $\left(C_{D \text { single }}=1,083\right)$. 


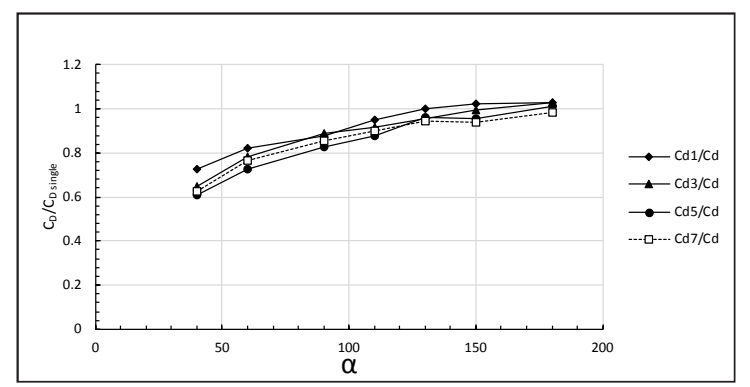

Fig. 17 drag coefficients for the spheres $1,3,5$ and $7, \mathrm{Re}=100$.

It is seen that for all spheres the drag increases with $\alpha$ and reach values close to this one of an isolated sphere. These parameters are influenced by the number of spheres and the angle of the configuration.

\section{Conclusions}

Hydrodynamic interaction of two or more rigid spherical particles in different arrangements have been studied numerically for moderate Reynolds numbers. It is confirmed that one of the most important factors acting on the flow parameters is the distance between the spheres. The strongest effect on the drag force when one or more spheres are included in the flow compared with a single sphere case arise for line arrangement where the difference could reach $12 \%$ and more. When particles are side by side due to zones with high and low pressure between them a weak repulsion and attraction forces appear. In the case of „V“ arrangement the angle $\alpha$ play significant role on the wake structure behind the particles and drag forces. The software used gives the possibility to study numerically with a high accuracy the flow past any formation of equal or different particles at different flow regimes.

\section{References}

1. G.G. Stokes, Trans. Camb. Phil. Soc. 9, 8, (1851)

2. M. Stimson, G.B. Jefferey, Proc. R. Soc. Lond A 111, 110-116, (1926)

3. S. Kim, Phys. Fluids, 30, 2309-2314, (1987)

4. H. Wilson, Journal of Computational Physics, 245, 302-316, (2013)

5. Y. Tsuji, Y. Morikawa, K. Terashima, Intl J. Multiphase Flow, 8 (1), 71-82, (1982)

6. C. Zhu, S. C Liang, L. S. Fan, Intl J. Multiphase Flow, 20 (1), 117-129, (1994)

7. R. C. Chen and J. L. Wu, Journal of Chem. Eng. Science, 55, 1143-1158, (2000)

8. I. Kim, S. Elghobashi, W. Sirignano, J . Fluid Mech., 246, 465-488, (1993).

9. L. Schouveiler, A. Brydon, T. Leweke, M.C. Thompson, European Journal of Mechanics B/Fluids 23, 137-145, (2004)

10. Van-Dyke, An Album of Fluid Motion, The parabolic Press, Stanford California, (1982)

11. Z. D. Zaprynov, E. T. Toshev, Proceedings of the Eighth International Conference on Heat Transfer, ASME, New York, 5, 2549-2553, (1986)

12. R. Tal, D. N Lee, W. A Sirignano, International Journal of Heat and Mass Transfer, 27, No. 11, 1953-1962, (1984)

13. R. Folkersma, H. Stein, F. Van de Vosse, International Journal of Multiphase Flow 26, 877-887, (2000) 